\title{
Human Factor Quantitative Analysis Based on OHFAM and Bayesian Network
}

\author{
Mei Rong ${ }^{1}$, Min Luo ${ }^{1}$, Yanqiu Chen ${ }^{1}$, Changhua Sun $^{2}$, and Yanyang Wang ${ }^{3}$ \\ ${ }^{1}$ China Academy of Civil Aviation Science and Technology, Beijing, China \\ \{rongmei, luomin, chenyq\} @mail.castc.org.cn \\ ${ }^{2}$ Civil Aviation Administration of China, Beijing, China \\ ch_sundcaac.gov.cn \\ ${ }^{3}$ Beihang University, Beijing, China \\ wangyanyang@buaa.edu.cn
}

\begin{abstract}
Occurrence Human Factors Analysis Model is developed for China civil aviation based on the Human Factors Analysis and Classification System (HFACS) and the actual operation conditions and characteristics of China civil aviation to enhance the classification, analysis and utilization of safety information. In addition, to generate a model which can provides quantitative analysis supportive in aviation incident analysis, a human factor analysis model based on OHFAM and Bayesian network is established. This model constructed by combing hill-climbing search method with $\mathrm{CH}$ score function is a Bayesian network which uses three layers nodes to represent causality between human factors and incidents. The specific impact degree of human factors on aviation incidents is represented by conditional probability parameters of the model. It is useful in aviation incident analyses and deductions.
\end{abstract}

Keywords: Occurrence Human Factors Analysis, Human factor quantitative analysis, Bayesian networks, Civil aviation safety, incident analysis.

\section{$1 \quad$ Introduction}

Statistic information of aviation accidents investigation shows that nearly $80 \%$ accidents were caused by human factors [1], and we also have got the point that we should not only concern about human error, but more in-depth study on the organization and management factors. According to Reason model, the failures of all levels interact and lead to disastrous consequences; the failures are the "holes" of system at different levels [2]. Human Factors Analysis and Classification System (HFACS) is developed based on Reason model [3], this model helps to define the "holes of cheese model". However, the existing human factors analysis model cannot fully meet China's actual needs. Therefore, we develop a model named "Occurrence Human Factors Analysis Model (OHFAM)" for China civil aviation human factors analysis and use the model to analyze the 180 crew caused incidents from 2001 to 2010 in China.

However, All these analysis models includes the OHFAM can hardly provide a more detailed and quantitative analysis [4]. This paper based on Bayesian network 
theory, employed the layer structure of OHFAM, developed human factor quantitative analysis model. By using this model to analyze civil aviation incidents statistic data, detailed impact degree of every human factor to incidents is derived as Bayesian network parameters to help diagnose civil aviation incidents.

\section{Occurrence Human Factors Analysis Model}

The OHFAM has five layers including "Unsafe Behavior", "Preconditions for Unsafe Behavior", "Department Management", "Organizational Influence" and "Government Supervision". The model clarifies its sub-categories of factors (as shown in Fig. 1) and gives various items as the expression of each factor.

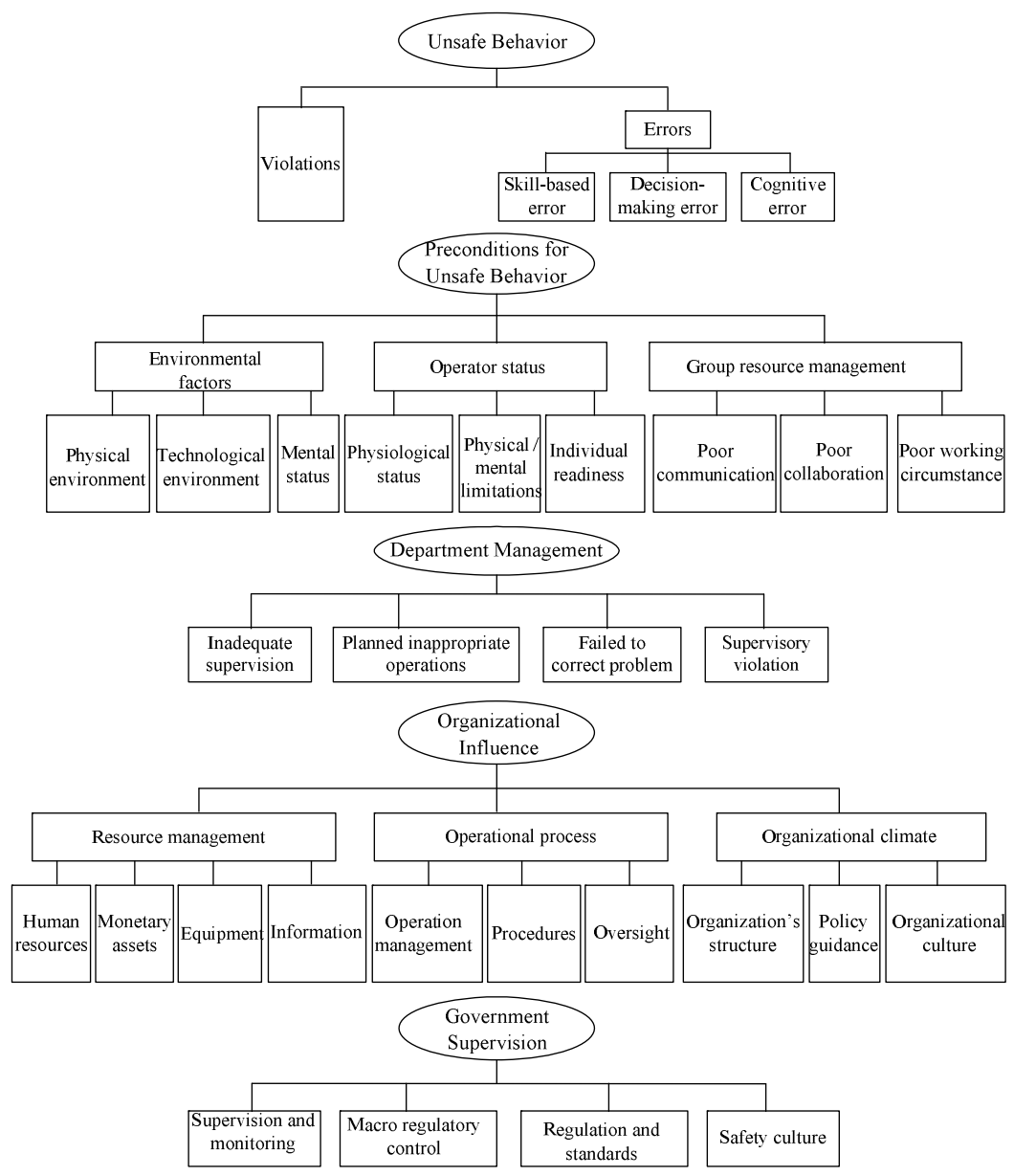

Fig. 1. OHFAM Framework 


\section{Bayesian Network}

A Bayesian network is a binary group $(S, P)$ in which $S$ stands for a directed acyclic graph and the node of the graph stands for stochastic variable, the edge between nodes stands for the dependency relationship between the variables [5]. $\mathrm{P}$ is conditional probability distribution $p\left(x_{i} \mid \pi\left(x_{i}\right)\right)$, which stands for the strength of dependency relationship among nodes. $\pi\left(x_{i}\right)$ is the set of parent nodes of $x_{i}$. A simple Bayesian network contains 5 nodes is described in Fig. 2.

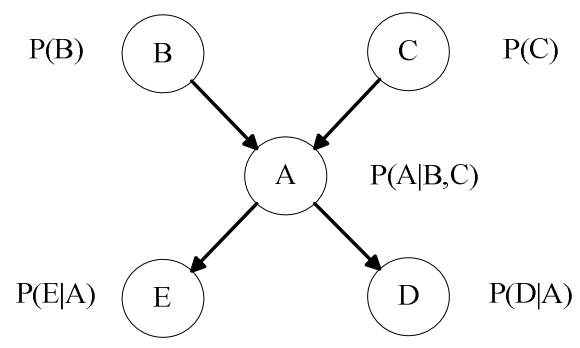

Fig. 2. A simple Bayesian network

The first step to construct a Bayesian network is to distinguish the key variables and its possible values of the domain in which the model will be built, and use nodes to stand for the variables, then to determine the dependency and independency relation between the nodes and express it in a graph. The final step is to calculate the ration part's probability parameter of the Bayesian network [6].Network construction is also called Bayesian network structure learning if both network structure and parameter are unknown. Generally, structure learning contains two steps: model selection and model optimization. Model selection refers to evaluate different model structure while model optimization is a searching process which aiming at searching the best model structure under the given sample data.

Given a data set $D=\left(D_{1}, D_{2}, \cdots, D_{m}\right)$, the good and bad of a specific network

structure $S$ can be measured by a score function. Bayesian network theory provides many kinds of score functions among which the most common used are $\mathrm{CH}$ score and BIC Score. Model optimization is a process of searching the best structure for the given sample data. The most common used searching algorithm in Bayesian network theory are $\mathrm{K} 2$ and hill climbing.

\section{Human Factor Quantitative Analysis Model Based on OHFAM and Bayesian Network}

The OHFAM, as is shown in Fig.1, has five layers. The layer 1 "Unsafe Behavior" is dominant factor while layer 2 to 5 are recessive factors. 
Human Factor Quantitative Analysis model we developed is based on Bayesian network theory and adopted the layered structure of OHFAM. In this paper, only layer 1 and layer 2 of OHFAM are employed to build the Human Factor Quantitative Analysis model, it contains three layers.

The Fig. 3 shows the structure of a Bayesian network analysis model. The network analysis model has three layers: preconditions for unsafe behavior, unsafe behavior, and incidents. In the network, each node stands for a variable which use two possible values, 1 or 0 , to represent whether the factor or incidents happened. A parameter is a conditional probability between two nodes in different layer, for example, the parameter of edge(Precondition3,Behavior2) is $P(B 2 \mid P 3)$ which stands for the probability of happing Behavior2 on condition of happing Precondition3.

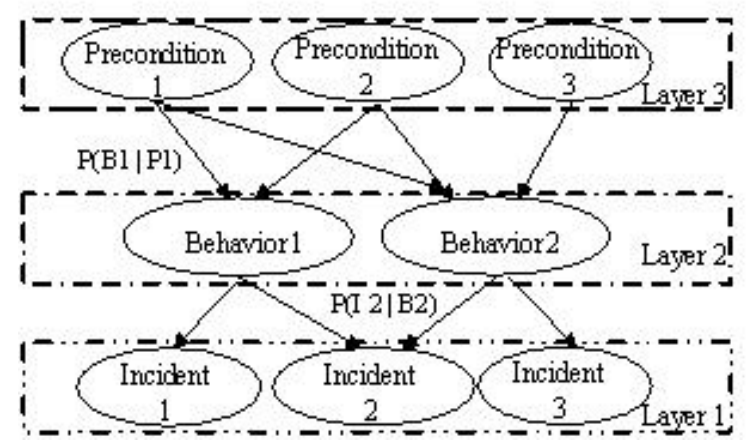

Fig. 3. Bayesian network human factor quantitative analysis model

Nodes in unsafe behavior precondition layer contains precondition nodes like: poor visibility, lack of cross validation, etc. Unsafe behaviors nodes like: misjudge on flight posture, violate flight rules, improper bias correction, etc.

As for Bayesian network theory, direct connection (edge) from nodes of layer 3 to layer 1 or within a layer is possible to generate in construction process. However, to combine Bayesian network theory with OHFAM, we changed the network search algorithm to confine the result network within a layered Bayesian network model which has no cross-layer or reverse connections.

Using the incidents database of China civil aviation, and taking the analysis results of the 180 crew caused incidents from 2001 to 2010 by OHFAM as the sample data of Bayesian network's structure learning and parameter learning, four networks are constructed by collocating $\mathrm{K} 2$ and hill climbing searching method with $\mathrm{BIC}$ and $\mathrm{CH}$ score functions. The agreement degree of each network compared with the actual data is calculated and listed in table 1. 
Table 1. Compare of Different Algorithm Collocation

\begin{tabular}{|l|c|c|l|}
\hline Algorithm collocation & Accuracy & Complexity & \multicolumn{1}{c|}{ Note } \\
\hline K2+CH & $87 \%$ & Middle & $\begin{array}{l}\text { This collocation has a large searching scope, each } \\
\text { behavior and premise will be searched. }\end{array}$ \\
\hline K2+BIC & $23 \%$ & Simple & $\begin{array}{l}\text { The accuracy of the network result under this } \\
\text { collocation is bad because the contradictory of the } \\
\text { two algorithms, so it's not a good construct collo- } \\
\text { cation. }\end{array}$ \\
\hline Hill Climbing +CH & $98 \%$ & Complex & $\begin{array}{l}\text { The result network is extremely complex because } \\
\text { hill climbing algorithm calculates each network } \\
\text { structure many times to get a more accurate } \\
\text { network. }\end{array}$ \\
\hline
\end{tabular}

\section{$5 \quad$ Human Factor Quantitative Analysis}

In this paper, network constructed under hill-climbing method and $\mathrm{CH}$ score is analyzed as an example for its accuracy, part of it is picked out and shown in Fig.4. (The whole network contains over 130 nodes and over 400 edges.)

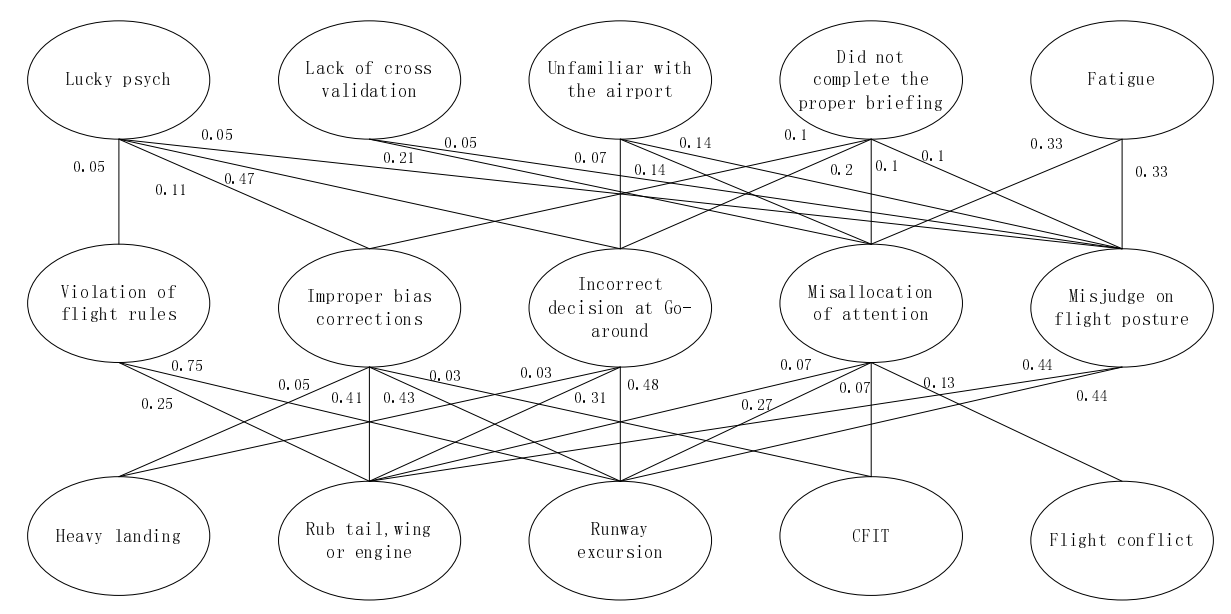

Fig. 4. Part of model network constructed by hill-climbing and BIC

According to the conditional probability parameter of the network, analyst can find out which human factors are the most possible ones that cause the specific incident. It is also available to know which incidents may occur as well as the probability of occurrence if a particular unsafe behavior has happened. Table 2 lists the incidents caused by incorrect decision at go-around (unsafe behavior) and the conditional probability. 
Table 2. Parameter Sample Between Layer 1 and Layer 2

\begin{tabular}{|l|c|l|c|}
\hline \multicolumn{4}{|c|}{ Unsafe behavior: Incorrect decision at go-around } \\
\hline \multicolumn{1}{|c|}{ Incident } & \multicolumn{1}{|c|}{ Incident } & Conditional probability & \multicolumn{1}{c|}{ probability } \\
\hline Heavy landing & 0.03 & Runway excursion & 0.48 \\
\hline Rub tail, wing or engine & 0.31 & Touch ground out of track & 0.18 \\
\hline
\end{tabular}

Table 3 lists the unsafe behaviors that maybe caused by fatigue (preconditions for unsafe behavior) and the conditional probability. The sum of all conditional probabilities caused by one particular node can be more than 1 because the results are not mutually exclusive to each other; they may happen at the same time.

Table 3. Parameter Sample Between Layer 2 and Layer 3

\begin{tabular}{|l|c|l|c|}
\hline \multicolumn{4}{|c|}{ Preconditions for unsafe behavior : Fatigue } \\
\hline Unsafe Behavior & $\begin{array}{c}\text { Conditional } \\
\text { probability }\end{array}$ & \multicolumn{1}{|c|}{ Unsafe Behavior } & $\begin{array}{c}\text { Conditional } \\
\text { probability }\end{array}$ \\
\hline Misjudge on flight posture & 0.33 & Improper landing, taking off decision & 0.33 \\
\hline Misallocation of attention & 0.33 & Crude and fierce operation & 0.33 \\
\hline
\end{tabular}

Conditional probability between layer 1 and layer 3 is also available from the result model network with the help of network parameters and statistic information. Table 4 lists the incidents that maybe caused by lucky psych and the conditional probability.

Table 4. Parameter Sample Between Layer 1 and Layer 3

\begin{tabular}{|l|c|l|c|}
\hline \multicolumn{4}{|c|}{ Unsafe behavior premise : Lucky psych } \\
\hline \multicolumn{1}{|c|}{ Incident } & Conditional probability & \multicolumn{1}{c|}{ Incident } & Conditional probability \\
\hline Runway excursion & 0.52 & Heavy landing & 0.01 \\
\hline Rub tail, wing or engine & 0.35 & CFIT & 0.01 \\
\hline Touch ground out of track & 0.09 & & \\
\hline
\end{tabular}

\section{Conclusion}

The human factor quantitative analysis model based on OHFAM and Bayesian Network established by combining hill climbing search method with $\mathrm{CH}$ score function, integrated the advantages of OHFAM and Bayesian network theory, can precisely reflect the causality between human factors and civil aviation incidents. The model uses conditional probability to represent the exact impact degree of human factors on incidents which is useful for incidents analysis and risk evaluation.

\section{References}

1. Guopeng, L.: Analysis of Accident Resulted from Human Factors in Safety Management. Journal of Civil Aviation University of China 27(4), 18-21 (2009)

2. Reason, J.: Human Error. Cambridge University Press, Cambridge (1990) 
3. Wiegmann, D.A., Shappell, S.A.: A Human Error Approach to Aviation Accident Analysis: The Human Factors Analysis and Classification System. Ashgate Publishing (2003) ISBN-13: 978-0754618737

4. Huiying, Z., Yuan, N.: Reasoning and Analysis of Bayesian Network in Machine Learning. Modern Machinery 2 (2012)

5. Lianwen, Z., Haipeng, G.: Bayesian network introduction. In: Structure Learning, pp. 172191. Science Press, Beijing (2006)

6. Cao, W., Lin, X.: Flight turnaround time analysis and delay prediction based on Bayesian network. Computer Engineering and Design 32(5), 1770-1773 (2001) 